\title{
Endometriosis and Recurrent Pregnancy Loss as New Risk Factors for Venous Thromboembolism during Pregnancy and Post-Partum: The JECS Birth Cohort
}

\author{
Mayumi Sugiura-Ogasawara ${ }^{1}$ Takeshi Ebara ${ }^{2}$ Taro Matsuki ${ }^{2}$ Yasuyuki Yamada ${ }^{2,3}$ Toyonori Omori ${ }^{4,5}$ \\ Yosuke Matsumoto $^{1}$ Sayaka Kato ${ }^{2,6}$ Hirohisa Kano ${ }^{2}$ Takahiro Kurihara ${ }^{2}$ Shinji Saitoh ${ }^{6}$ \\ Michihiro Kamijima ${ }^{2}$ and the Japan Environment \& Children's Study (JECS) Group
}

${ }^{1}$ Department of Obstetrics and Gynecology, Graduate School of Medical Sciences, Nagoya City University, Nagoya, Japan

2 Department of Occupational and Environmental Health, Graduate School of Medical Sciences, Nagoya City University, Nagoya, Japan

${ }^{3}$ Graduate School of Health and Sports Science, Juntendo University, Chiba, Japan

${ }^{4}$ Department of Health Care Policy and Management, Graduate School of Medical Sciences, Nagoya City University, Nagoya, Japan

${ }^{5}$ National Center for Child Health and Development, Tokyo, Japan

6 Department of Pediatrics and Neonatology, Graduate School of Medical Sciences, Nagoya City University, Nagoya, Japan
Address for correspondence Mayumi Sugiura-Ogasawara, MD, PhD, Department of Obstetrics and Gynecology, Graduate School of Medical Science, Nagoya City University, Mizuho-ku, Nagoya 4678601, Japan (e-mail: og.mym@med.nagoya-cu.ac.jp).

Thromb Haemost 2019;119:606-617.

\begin{abstract}
Keywords

- venous thromboembolism

- birth cohort study

- pregnancy

- post-partum

- recurrent pregnancy loss
\end{abstract}

Background Since venous thromboembolism (VTE) is one of the causes of maternal mortality, several guidelines recommend prophylaxis using low molecular weight heparin for women in high-risk groups. The number of large population-based studies examining predictors for VTE has been limited, and there has been no study based on a Japanese population.

Objective Our objective was to examine VTE risk factor during the pregnancy and post-partum period.

Materials and Methods A nationwide birth cohort study known as the 'Japan Environment and Children's Study (JECS)' was conducted by the Ministry of the Environment. The subjects consisted of 103,070 pregnancies recruited by the JECS between January 2011 and March 2014. Pregnant women completed the questionnaires during the first and second/ third trimester. Their medical records were transcribed by physicians or research coordinators at registration, just after delivery and at 1 month after delivery.

Results The frequency of VTE was 7.5 per 10,000 pregnancies $(77$ of 103,070 ) during the pregnancy and post-partum period. After the adjustment of multiple covariates for each factor, endometriosis and recurrent pregnancy loss (RPL) were identified as novel independent risk factors for VTE. Adjusted odds ratios were as follows: 2.70 (95\% confidence interval, 1.21-6.00) for endometriosis and 6.13 (2.48-15.16) for RPL. Threatened abortion, threatened pre-term birth, pre-term birth and caesarean section were ascertained to be risk factors for VTE.

Conclusion Careful attention should be given to novel predictors, such as endometriosis and a history of RPL, to prevent VTE during the pregnancy and post-partum period. received

October 11, 2018

accepted after revision

December 17, 2018
DOI https://doi.org/

$10.1055 / \mathrm{s}-0039-1677733$.

ISSN 0340-6245. (c) 2019 Georg Thieme Verlag KG Stuttgart · New York
License terms

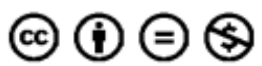




\section{Introduction}

It is well known that pregnancy and puerperium is associated with venous thromboembolism (VTE). The frequency of post-partum VTE was reported to be 7.2 per 10,000 deliveries in an English cohort registered between 1997 and 2014 and 7.9 in a Swedish cohort registered between 2005 and 2011. ${ }^{1}$ Since VTE is one of the causes of maternal mortality, several guidelines recommend prophylaxis using low molecular weight heparin (LMWH) for women in the high-risk group. ${ }^{2,3}$ The strongest risk factor is a previous episode. ${ }^{2,3}$ The recurrence rate of withholding heparin was $2.4 \%$ in 125 pregnant women with previous VTE, $0 \%$ in 44 women without thrombophilia and $5.9 \%$ in 51 women with thrombophilia and/or previous episode of idiopathic thrombosis. ${ }^{4}$ Pregnant women who have had two or more episodes, one episode plus thrombophilia associated with anti-phospholipid antibodies, protein $\mathrm{C}(\mathrm{PC})$, protein $\mathrm{S}$ (PS) or an antithrombin (AT) deficiency, and one episode plus a history of VTE in a first-degree relative are all included in the highest risk group. ${ }^{2,3}$ The second highest risk group includes women with an isolated episode during bed rest, dehydration and surgery, thrombophilia or with a complication such as heart disease, lung disease, systemic lupus erythematosus (SLE), cancer, inflammatory bowel disease or nephrotic syndrome. ${ }^{2,3,5}$ The third highest group includes women with an age $\geqq 35$ years, a body mass index (BMI) of $>30 \mathrm{~kg} / \mathrm{m}^{2}$, a smoker, parity $\geqq 3$, a systemic infection, gross varicose veins, paraplegia, pre-eclampsia, hyperemesis, ovarian hyperstimulation syndrome, multiple pregnancies, use of artificial reproductive technology, caesarean section and post-partum haemorrhage. 2,3,5 Accordingly, LMWH is recommended for pregnant women with three or more of these risk factors.

However, both the Royal College of Obstetricians and Gynaecologists (RCOG) guidelines and the American College of Chest Physicians (ACCP) evidence-based clinical practice guidelines have recommended that LMWH use should be decided upon after discussion between two or more doctors in the case of the second and third highest risk groups since these guidelines were based on expert opinion due to limited evidence concerning the effect of thromboprophylaxis during pregnancy. $2,3,6$

The Japan Society of Obstetrics and Gynecology (JSOG) drew up guidelines in 2014 that conformed to the RCOG and ACCP guidelines due to a lack of evidence in Japan. ${ }^{7}$ Before April 2014, thromboprophylaxis was performed according to the previous guidelines, under which an episode of VTE, thrombophilia, age $\geqq 35$ years, a caesarean section and obesity were considered to be risk factors for Japanese women. ${ }^{8}$ For the highest risk group, low dose unfractionated heparin was recommended for women with a past episode of VTE or thrombophilia who underwent a caesarean section during the post-partum period. Unfractionated heparin or intermittent pneumatic compression was recommended for older obese women after caesarean section or for women with an episode of VTE or thrombophilia after vaginal delivery in the high-risk group. There has been no study of VTE risk factors in Japanese women during the pregnancy and post-partum period. Therefore, it was considered necessary to examine these factors because the frequency of VTE is smaller in Japanese populations than in Caucasian population and the frequency of thrombophilia depends on race. ${ }^{9}$

We have conducted a nationwide population-based birth cohort study known as the 'Japan Environment and Children's Study (JECS)', a project planned by the Ministry of the Environment, Government of Japan. ${ }^{10-14}$ The study subjects consisted of 104,102 registered children or foetuses recruited during the first 3 years of the JECS, and the babies are now being followed up for 13 years mainly to examine the influence of the environment on the foetus.

This is the first birth cohort used to examine the frequency of VTE and to determine VTE risk factors during the pregnancy and post-partum period in a Japanese population.

\section{Materials and Methods}

\section{Study Design and Participants}

Pregnant women were recruited by the JECS between January 2011 and March 2014. Eligibility criteria for expectant mothers were as follows: that they (1) resided at the time of recruitment in any of the study areas selected by 15 regional JECS centres located countrywide; (2) had an expected delivery date after August 1, 2011; and (3) were capable of comprehending the Japanese language and completing the self-administered questionnaire. ${ }^{10-14}$ The sample size has been calculated in the JECS protocol by the Ministry of the Environment. ${ }^{15}$ In principle, pregnant women completed the questionnaires during the first (MT1) and second/third trimester (MT2). Their medical records were transcribed by physicians or research coordinators at registration (DrT1), just after delivery (Dr0m) and at 1 month after delivery (Dr1m).

This study was based on the jecs-ag-20160424 dataset, which includes 104,102 registered children (foetuses and embryos), and was released restrictively to all concerned in June 2016. The second and third children of multiple pregnancies were excluded and these numbered 1,003 (0.96\%). Twenty-nine participants $(0.03 \%)$ withdrew their consent completely. Finally, 103,070 pregnancies were included in the main analysis. The mean (standard deviation [SD]) age at registration was 30.7 (5.1) years. The mean (SD) gestational weeks at registration was 14.0 (5.7) weeks. The JECS population has been recognized as representative of the pregnant women in Japan. ${ }^{11}$

The JECS protocol was reviewed and approved by the Ministry of the Environment's Institutional Review Board on Epidemiological Studies and by the Ethics Committees of all participating institutions. Written informed consent was obtained from all participating women.

\section{Data Collection}

The first questionnaire (MT1) included socio-demographic characteristics, medical histories, the details of all previous pregnancies and exercise habits.

The socio-economic status was assessed by the education level and annual household income in the second questionnaire (MT2). The MT2 included lifestyle details. 
The first medical record transcript (DrT1) included maternal age, gestational weeks at registration, maternal body weight, height, conception and details of all previous pregnancies (vaginal delivery/caesarean delivery/miscarriage/ induced abortion/stillbirth).

The Dr0m included maternal age, gestational weeks at miscarriage and delivery, single/multiple pregnancies, live birth/stillbirth, miscarriage/induced abortion, male/female, birth weight, vaginal/caesarean delivery, pregnancy complications and perinatal outcome.

VTE was described in the Dr0m and in the third medical record transcription (Dr1m).

\section{Exposures and Covariates}

Potential exposures were compiled from medical and obstetrical histories as well as from information on gynaecological diseases, obstetric complications and lifestyle.

Potential covariates were maternal age at registration (categorized as $<20,20-29,30-39, \geqq 40$ years), BMI (categorized as $<18.5,18.5-25.0, \geqq 25.0$ ), the presence/absence of in vitro fertilization and embryo transfer (IVF-ET), smoking status and income level (categorize as $<200,200-<400$, $400-<600,600-<800,800-<1,000$ JPY $\times 10,000$ [1 US $\$=114.66 \mathrm{JPY}$, November 13,2018$]$ ).

\section{Statistical Analysis}

Details of potential exposures are listed in - Supplementary Table S1 (available in the online version).

Frequencies for discrete data between VTE and each factor were counted with proportion. To compare their respective distributions, a Fisher's exact test was performed. Crude odds ratios (ORs) for all exposures were calculated and multiple logistic regression analyses were further conducted to determine what kind of exposures was predictive of VTE after controlling for maternal age, BMI, the presence/absence of IVF-ET, smoking status and income level. In case the observed number of each factor and VTE was less than 6 , adjusted ORs were not calculated because of potential biases depending on the number of cases in the less frequencies.

All calculations were conducted using SPSS version 23 and 24 (IBM Corp., Japan), and a $p$-value of $<0.05$ was regarded as statistically significant.

\section{Results}

The frequency of VTE was 7.5 per 10,000 pregnancies (77 of $103,070)$ during the pregnancy and post-partum period. The presence/absence of VTE according to each factor is shown in -Tables 1 and 2. Age, BMI, the presence of IVF-ET, smoking status and income level were significantly associated with VTE.

After the adjustment of multiple covariates for each factor, endometriosis and recurrent pregnancy loss (RPL) were judged to be novel independent predictors for VTE (-Table 3). Adjusted ORs were as follows: 2.70 (95\% confidence interval [CI], 1.21-6.00) for endometriosis and 6.13 (2.48-15.16) for RPL. There was no statistically significant risk related to any of the listed medical histories or lifestyle other than those mentioned above.
Regarding pregnancy complications and outcomes, oligohydramnios might be a novel independent predictor for VTE though adjusted ORs were not shown since the number was less than 6 (-Table 4). Threatened abortion, threatened preterm birth, pre-term birth at $<37$ weeks and caesarean section remained significantly associated with VTE. Adjusted ORs were as follows: 3.61 (95\% CI, 2.16-6.02) for threatened abortion, 2.98 (1.83-4.86) for threatened pre-term birth, $2.64(1.30-5.36)$ for pre-term birth both at $<37$ weeks and 2.19 (1.32-3.63) for caesarean section.

There was no association with hyperemesis or mild hypertensive disorders of pregnancy (HDP) with VTE. The association with a history of placenta praevia, adenomyosis and PCOS, pre-term birth $<34$ weeks' gestation, severe HDP, placenta praevia, abruptio placenta, multiple pregnancy and VTE were unclear in this study because the sample size was relatively small. There were no cases of VTE in patients with a history of stroke, myocardial infarction, congenital heart disease, SLE, Crohn's disease, ulcerative colitis, nephrotic syndrome or various forms of cancer.

\section{Discussion}

In the present cohort, we found endometriosis and three or more pregnancy losses as novel risk factors for VTE.

Endometriosis is characterized by endometrial-like tissue outside the uterine and is a major contributor to pelvic pain and infertility. ${ }^{16}$ This is the first study that we know of to show that endometriosis is a VTE risk factor. In fact, there was one study in which no episode of deep vein thrombosis and VTE occurred after laparoscopic surgery in a group of 266 patients in which $21 \%$ had endometriosis. ${ }^{17}$ Lesions and activated macrophages can secrete pro-inflammatory cytokines such as interleukin-1 $\beta$, interleukin- 6 , interleukin- 8 and tumour necrosis factor $\alpha$ in the peritoneal cavity of patients with endometriosis. ${ }^{16}$ These pro-inflammatory cytokines might trigger VTE during pregnancy.

With regard to RPL, to our knowledge, this is the first study to show that a history of RPL has a direct predictive value for assessing VTE risk. Stillbirth was not included in eight women with both a history of RPL and VTE, though stillbirth is well-known to be a strong risk factor for postpartum VTE. ${ }^{1}$ RPL and VTE are considered to be associated with acquired and inheritable thrombophilia such as AT deficiency, PC deficiency, PS deficiency and homozygous factor V (FV) Leiden. ${ }^{18}$ Clinical criteria for anti-phospholipid syndrome (APS) include thrombosis, recurrent miscarriage, intrauterine foetal death and early-onset pre-term birth following pre-eclampsia or placental insufficiency. ${ }^{19}$ Patients with APS received combined therapy of low dose aspirin and heparin, and this applied to 162 women in the present cohort with APS. RPL was an independent predictor when APS was used as a covariate (not shown). Recently, 472 variants in 187 genes have been reported to be associated with RPL. ${ }^{20}$ A meta-analysis revealed a significant association between RPL and 21 variants, including loss due to thrombophilia with ORs of 0.51 to 2.37. Thrombophilia related to FV Leiden mutation, pro-thrombin mutation, 
New Risk Factors for VTE during Pregnancy and Post-Partum Sugiura-Ogasawara et al. 609

Table 1 The presence/absence of venous thromboembolism according to each factor

\begin{tabular}{|c|c|c|c|c|c|c|c|}
\hline & \multirow[t]{2}{*}{ Factors } & \multirow{2}{*}{$\begin{array}{l}N \\
\text { (Missing data) }\end{array}$} & \multirow[t]{2}{*}{$\%$} & & \multicolumn{2}{|c|}{$\begin{array}{l}\text { Venous } \\
\text { thromboembolism }\end{array}$} & \multirow[t]{2}{*}{$p$-Value ${ }^{a}$} \\
\hline & & & & & Absence & Presence & \\
\hline \multirow{44}{*}{$\begin{array}{l}\text { Medical } \\
\text { histories }\end{array}$} & \multirow[t]{2}{*}{ Atopic dermatitis } & 98,766 & 95.8 & Absence & 83,178 & 60 & \multirow[t]{2}{*}{0.207} \\
\hline & & $(4,304)$ & $(4.2)$ & Presence & 15,512 & 16 & \\
\hline & \multirow[t]{2}{*}{ Asthma } & 98,766 & 95.8 & Absence & 87,905 & 64 & \multirow[t]{2}{*}{0.194} \\
\hline & & $(4,304)$ & $(4.2)$ & Presence & 10,785 & 12 & \\
\hline & \multirow[t]{2}{*}{ Collagen disease } & 98,766 & 95.8 & Absence & 98,560 & 76 & \multirow[t]{2}{*}{-} \\
\hline & & $(4,304)$ & $(4.2)$ & Presence & 130 & 0 & \\
\hline & \multirow[t]{2}{*}{ Autoimmune disease } & 98,766 & 95.8 & Absence & 98,548 & 76 & \multirow[t]{2}{*}{-} \\
\hline & & $(4,304)$ & $(4.2)$ & Presence & 142 & 0 & \\
\hline & \multirow[t]{2}{*}{ SLE } & 98,766 & 95.8 & Absence & 98,618 & 76 & \multirow[t]{2}{*}{-} \\
\hline & & $(4,304)$ & $(4.2)$ & Presence & 72 & 0 & \\
\hline & \multirow[t]{2}{*}{ RA } & 98,766 & 95.8 & Absence & 98,483 & 76 & \multirow[t]{2}{*}{-} \\
\hline & & $(4,304)$ & $(4.2)$ & Presence & 207 & 0 & \\
\hline & \multirow[t]{2}{*}{ IDDM } & 98,766 & 95.8 & Absence & 98,614 & 76 & \multirow[t]{2}{*}{-} \\
\hline & & $(4,304)$ & $(4.2)$ & Presence & 76 & 0 & \\
\hline & \multirow[t]{2}{*}{ NIDDM } & 98,766 & 95.8 & Absence & 98,556 & 76 & \multirow[t]{2}{*}{-} \\
\hline & & $(4,304)$ & $(4.2)$ & Presence & 134 & 0 & \\
\hline & Gestational diabetes & 98,766 & 95.8 & Absence & 97,950 & 75 & 0.436 \\
\hline & & $(4,304)$ & $(4.2)$ & Presence & 740 & 1 & \\
\hline & Hyperthyroidism & 98,766 & 95.8 & Absence & 97,635 & 76 & - \\
\hline & & $(4,304)$ & $(4.2)$ & Presence & 1,055 & 0 & \\
\hline & Hypothyroidism & 98,766 & 95.8 & Absence & 97,714 & 75 & 0.530 \\
\hline & & $(4,304)$ & $(4.2)$ & Presence & 976 & 1 & \\
\hline & Anaemia & 98,766 & 95.8 & Absence & 80,446 & 60 & 0.555 \\
\hline & & $(4,304)$ & $(4.2)$ & Presence & 18,244 & 16 & \\
\hline & Hypertension & 98,766 & 95.8 & Absence & 98,220 & 76 & - \\
\hline & & $(4,304)$ & $(4.2)$ & Presence & 470 & 0 & \\
\hline & Hyperlipidaemia & 98,766 & 95.8 & Absence & 98,206 & 76 & - \\
\hline & & $(4,304)$ & $(4.2)$ & Presence & 484 & 0 & \\
\hline & Stroke & 98,766 & 95.8 & Absence & 98,579 & 76 & - \\
\hline & & $(4,304)$ & $(4.2)$ & Presence & 111 & 0 & \\
\hline & Myocardial infarction & 98,766 & 95.8 & Absence & 98,628 & 76 & - \\
\hline & & $(4,304)$ & $(4.2)$ & Presence & 62 & 0 & \\
\hline & Congenital heart disease & 98,766 & 95.8 & Absence & 98,387 & 76 & - \\
\hline & & $(4,304)$ & $(4.2)$ & Presence & 303 & 0 & \\
\hline & Kawasaki disease & 98,766 & 95.8 & Absence & 98,271 & 76 & - \\
\hline & & $(4,304)$ & $(4.2)$ & Presence & 419 & 0 & \\
\hline & Depression & 98,766 & 95.8 & Absence & 95,701 & 72 & 0.295 \\
\hline & & $(4,304)$ & $(4.2)$ & Presence & 2,989 & 4 & \\
\hline & Dysautonomia & 98,766 & 95.8 & Absence & 95,044 & 72 & 0.366 \\
\hline & & $(4,304)$ & $(4.2)$ & Presence & 3,646 & 4 & \\
\hline & Anxiety disorder & 98,766 & 95.8 & Absence & 95,904 & 71 & 0.064 \\
\hline & & $(4,304)$ & $(4.2)$ & Presence & 2,786 & 5 & \\
\hline & Gastritis & 98,766 & 95.8 & Absence & 90,166 & 65 & 0.097 \\
\hline & & $(4,304)$ & $(4.2)$ & Presence & 8,524 & 11 & \\
\hline
\end{tabular}


Table 1 (Continued)

\begin{tabular}{|c|c|c|c|c|c|c|c|}
\hline & \multirow[t]{2}{*}{ Factors } & \multirow{2}{*}{$\begin{array}{l}N \\
\text { (Missing data) }\end{array}$} & \multirow[t]{2}{*}{$\%$} & & \multicolumn{2}{|c|}{$\begin{array}{l}\text { Venous } \\
\text { thromboembolism }\end{array}$} & \multirow[t]{2}{*}{$p$-Value ${ }^{a}$} \\
\hline & & & & & Absence & Presence & \\
\hline & \multirow[t]{2}{*}{ Gastric ulcer } & 98,766 & 95.8 & Absence & 96,998 & 73 & \multirow[t]{2}{*}{0.142} \\
\hline & & $(4,304)$ & $(4.2)$ & Presence & 1,692 & 3 & \\
\hline & \multirow[t]{2}{*}{ Irritable colon } & 98,766 & 95.8 & Absence & 97,152 & 75 & \multirow[t]{2}{*}{1.000} \\
\hline & & $(4,304)$ & $(4.2)$ & Presence & 1,538 & 1 & \\
\hline & \multirow[t]{2}{*}{ Crohn's disease } & 98,766 & 95.8 & Absence & 98,655 & 76 & \multirow[t]{2}{*}{-} \\
\hline & & $(4,304)$ & $(4.2)$ & Presence & 35 & 0 & \\
\hline & \multirow[t]{2}{*}{ Ulcerative colitis } & 98,766 & 95.8 & Absence & 98,468 & 76 & \multirow[t]{2}{*}{-} \\
\hline & & $(4,304)$ & $(4.2)$ & Presence & 222 & 0 & \\
\hline & \multirow[t]{2}{*}{ Fatty liver } & 98,766 & 95.8 & Absence & 98,446 & 76 & \multirow[t]{2}{*}{-} \\
\hline & & $(4,304)$ & $(4.2)$ & Presence & 244 & 0 & \\
\hline & \multirow[t]{2}{*}{ Chronic nephritis } & 98,766 & 95.8 & Absence & 98,349 & 76 & \multirow[t]{2}{*}{-} \\
\hline & & $(4,304)$ & $(4.2)$ & Presence & 341 & 0 & \\
\hline & \multirow[t]{2}{*}{ Nephrotic syndrome } & 98,766 & 95.8 & Absence & 98,599 & 76 & \multirow[t]{2}{*}{-} \\
\hline & & $(4,304)$ & $(4.2)$ & Presence & 91 & 0 & \\
\hline & \multirow[t]{2}{*}{ Breast cancer } & 98,766 & 95.8 & Absence & 98,635 & 76 & \multirow[t]{2}{*}{-} \\
\hline & & $(4,304)$ & $(4.2)$ & Presence & 55 & 0 & \\
\hline & \multirow[t]{2}{*}{ Cervical cancer } & 98,766 & 95.8 & Absence & 97,883 & 75 & 0.464 \\
\hline & & $(4,304)$ & $(4.2)$ & Presence & 807 & 1 & \\
\hline & Endometrial cancer & 98,766 & 95.8 & Absence & 98,682 & 76 & - \\
\hline & & $(4,304)$ & $(4.2)$ & Presence & 8 & 0 & \\
\hline & Gastric cancer & 98,766 & 95.8 & Absence & 98,687 & 76 & - \\
\hline & & $(4,304)$ & $(4.2)$ & Presence & 3 & 0 & \\
\hline & Colorectal cancer & 98,766 & 95.8 & Absence & 98,679 & 76 & - \\
\hline & & $(4,304)$ & $(4.2)$ & Presence & 11 & 0 & \\
\hline & Blood cancer & 98,766 & 95.8 & Absence & 98,650 & 76 & - \\
\hline & & $(4,304)$ & $(4.2)$ & Presence & 40 & 0 & \\
\hline & Other cancers & 98,766 & 95.8 & Absence & 98,515 & 76 & - \\
\hline & & $(4,304)$ & $(4.2)$ & Presence & 175 & 0 & \\
\hline Pregnancy & Pregnancy loss & 99,687 & 96.7 & Absence & 76,885 & 50 & 0.012 \\
\hline histories & (total number) & $(3,383)$ & (3.3) & Presence & 22,725 & 27 & \\
\hline & Pregnancy loss (once) & 94,645 & 91.8 & Absence & 76,885 & 50 & 0.343 \\
\hline & & $(8,425)$ & $(8.2)$ & Presence & 17,695 & 15 & \\
\hline & Pregnancy loss (twice) & 80,842 & 78.4 & Absence & 76,885 & 50 & 0.333 \\
\hline & & $(22,228)$ & $(21.6)$ & Presence & 3,903 & 4 & \\
\hline & Pregnancy loss & 78,070 & 75.7 & Absence & 76,885 & 50 & $<0.001$ \\
\hline & (three tımes or more) & $(25,000)$ & $(24.3)$ & Presence & 1,127 & 8 & \\
\hline & Hypertensive disorders & 98,766 & 95.8 & Absence & 96,791 & 76 & - \\
\hline & of pregnancy & $(4,304)$ & $(4.2)$ & Presence & 1,899 & 0 & \\
\hline & Gestational diabetes & 98,766 & 95.8 & Absence & 98,223 & 76 & - \\
\hline & & $(4,304)$ & $(4.2)$ & Presence & 467 & 0 & \\
\hline & Abruptio placentae & 98,766 & 95.8 & Absence & 98,455 & 75 & 0.166 \\
\hline & & $(4,304)$ & $(4.2)$ & Presence & 235 & 1 & \\
\hline & Ectopic pregnancy & 98,766 & 95.8 & Absence & 97,742 & 75 & 0.520 \\
\hline & & $(4,304)$ & $(4.2)$ & Presence & 948 & 1 & \\
\hline
\end{tabular}


Table 1 (Continued)

\begin{tabular}{|c|c|c|c|c|c|c|c|}
\hline & \multirow[t]{2}{*}{ Factors } & \multirow{2}{*}{$\begin{array}{l}N \\
\text { (Missing data) }\end{array}$} & \multirow[t]{2}{*}{$\%$} & & \multicolumn{2}{|c|}{$\begin{array}{l}\text { Venous } \\
\text { thromboembolism }\end{array}$} & \multirow[t]{2}{*}{$p$-Value ${ }^{a}$} \\
\hline & & & & & Absence & Presence & \\
\hline & \multirow[t]{2}{*}{ Placenta praevia } & 98,766 & 95.8 & Absence & 98,216 & 74 & \multirow[t]{2}{*}{0.052} \\
\hline & & $(4,304)$ & $(4.2)$ & Presence & 474 & 2 & \\
\hline & \multirow[t]{2}{*}{ Hydatidiform mole } & 98,766 & 95.8 & Absence & 98,262 & 76 & \multirow[t]{2}{*}{-} \\
\hline & & $(4,304)$ & $(4.2)$ & Presence & 428 & 0 & \\
\hline \multirow{14}{*}{$\begin{array}{l}\text { Gynaecological } \\
\text { disease }\end{array}$} & \multirow[t]{2}{*}{ Menstrual disorder } & 98,766 & 95.8 & Absence & 87,529 & 68 & \multirow[t]{2}{*}{1.000} \\
\hline & & $(4,304)$ & $(4.2)$ & Presence & 11,161 & 8 & \\
\hline & \multirow[t]{2}{*}{ Endometriosis } & 98,766 & 95.8 & Absence & 95,088 & 69 & \multirow[t]{2}{*}{0.021} \\
\hline & & $(4,304)$ & $(4.2)$ & Presence & 3,602 & 7 & \\
\hline & \multirow[t]{2}{*}{ Uterine fibroids } & 98,766 & 95.8 & Absence & 92,671 & 67 & \multirow[t]{2}{*}{0.049} \\
\hline & & $(4,304)$ & $(4.2)$ & Presence & 6,019 & 9 & \\
\hline & \multirow[t]{2}{*}{ Adenomyosis } & 98,766 & 95.8 & Absence & 98,355 & 74 & \multirow[t]{2}{*}{0.028} \\
\hline & & $(4,304)$ & $(4.2)$ & Presence & 335 & 2 & \\
\hline & \multirow[t]{2}{*}{ Uterine anomaly } & 98,766 & 95.8 & Absence & 98,408 & 76 & \multirow[t]{2}{*}{-} \\
\hline & & $(4,304)$ & $(4.2)$ & Presence & 282 & 0 & \\
\hline & \multirow[t]{2}{*}{ Ovarian tumour } & 98,766 & 95.8 & Absence & 95,267 & 74 & \multirow[t]{2}{*}{1.000} \\
\hline & & $(4,304)$ & $(4.2)$ & Presence & 3,423 & 2 & \\
\hline & \multirow{2}{*}{$\begin{array}{l}\text { Polycystic ovarian } \\
\text { syndrome }\end{array}$} & 98,766 & 95.8 & Absence & 96,477 & 71 & \multirow[t]{2}{*}{0.028} \\
\hline & & $(4,304)$ & $(4.2)$ & Presence & 2,213 & 5 & \\
\hline Lifestyle & Strong exercise & 96,753 & 93.9 & No & 93,448 & 72 & 0.325 \\
\hline & & $(6,317)$ & $(6.1)$ & Yes & 3,229 & 4 & \\
\hline & Moderate exercise & 96,091 & 93.2 & No & 72,207 & 64 & 0.049 \\
\hline & durıng pregnancy & $(6,979)$ & $(6.8)$ & Yes & 23,809 & 11 & \\
\hline & Walking during & 95,424 & 92.6 & No & 27,018 & 25 & 0.298 \\
\hline & pregnancy & $(7,646)$ & $(7.4)$ & Yes & 68,333 & 48 & \\
\hline & Night shift work & 97,035 & 94.1 & No & 88,910 & 70 & 1.000 \\
\hline & auring pregnancy & $(6,035)$ & $(5.9)$ & Yes & 8,049 & 6 & \\
\hline & Breakfast during & 97,064 & 94.2 & Everyday & 69,100 & 58 & 0.374 \\
\hline & pregnancy & $(6,006)$ & $(5.8)$ & Not everyday & 27,888 & 18 & \\
\hline & Working h/wk (MT1) & 59,428 & 57.7 & $0 \mathrm{~h}$ & 37,614 & 28 & 0.995 \\
\hline & & $(43,642)$ & $(42.3)$ & $0-35 \mathrm{~h}$ & 21,770 & 16 & \\
\hline & Working h/wk (MT1) & 63,449 & 61.6 & $0 \mathrm{~h}$ & 37,614 & 28 & 0.878 \\
\hline & & $(39,621)$ & $(38.4)$ & $36-45 \mathrm{~h}$ & 25,789 & 18 & \\
\hline & Working h/wk (MT1) & 49,807 & 48.3 & $0 \mathrm{~h}$ & 37,614 & 28 & 0.277 \\
\hline & & $(53,263)$ & $(51.7)$ & $>46 \mathrm{~h}$ & 12,152 & 13 & \\
\hline & Working h/wk (MT2) & 64,615 & 62.7 & $0 \mathrm{~h}$ & 45,672 & 33 & 1.000 \\
\hline & & $(38,455)$ & $(37.3)$ & $0-35 \mathrm{~h}$ & 18,896 & 14 & \\
\hline & Working h/wk (MT2) & 68,246 & 66.2 & $0 \mathrm{~h}$ & 45,672 & 33 & 0.663 \\
\hline & & $(34,824)$ & $(33.8)$ & $36-45 \mathrm{~h}$ & 22,522 & 19 & \\
\hline & Working h/wk (MT2) & 55,240 & 53.6 & $0 \mathrm{~h}$ & 45,672 & 33 & 0.311 \\
\hline & & $(47,830)$ & $(46.4)$ & $>46 \mathrm{~h}$ & 9,525 & 10 & \\
\hline
\end{tabular}

Abbreviations: IDDM, insulin-dependent diabetes mellitus; MT1, maternal questionnaires during the first trimester; MT2, maternal questionnaires during the second/third trimester; NIDDM, non-insulin-dependent diabetes mellitus; RA, rheumatoid arthritis; SLE, systemic lupus erythematosus. ${ }^{a}$ The $p$-value was not calculated when expected frequency is less than 1 . 
612 New Risk Factors for VTE during Pregnancy and Post-Partum Sugiura-Ogasawara et al.

Table 2 The presence/absence of venous thromboembolism according to each factor

\begin{tabular}{|c|c|c|c|c|c|c|}
\hline \multirow[t]{2}{*}{ Factors } & \multirow{2}{*}{$\begin{array}{l}N \\
\text { (Missing data) }\end{array}$} & \multirow[t]{2}{*}{$\%$} & & \multicolumn{2}{|c|}{$\begin{array}{l}\text { Venous } \\
\text { thromboembolism }\end{array}$} & \multirow[t]{2}{*}{ p-Value ${ }^{\mathrm{d}}$} \\
\hline & & & & Absence & Presence & \\
\hline \multirow[t]{2}{*}{ Threatened abortion } & 100,818 & 97.8 & Absence & 88,624 & 52 & \multirow[t]{2}{*}{$<0.001$} \\
\hline & $(2,252)$ & $(2.2)$ & Presence & 12,117 & 25 & \\
\hline \multirow[t]{2}{*}{ Threatened pre-term labour } & 100,818 & 97.8 & Absence & 80,909 & 47 & \multirow[t]{2}{*}{$<0.001$} \\
\hline & $(2,252)$ & $(2.2)$ & Presence & 19,832 & 30 & \\
\hline \multirow[t]{2}{*}{ Hyperemesis } & 97,070 & 94.2 & Absence & 16,745 & 11 & \multirow[t]{2}{*}{0.648} \\
\hline & $(6,000)$ & $(5.8)$ & Presence & 80,249 & 65 & \\
\hline \multirow[t]{2}{*}{ Early miscarriage $(<12 \text { wk gestation })^{a}$} & 99,614 & 96.6 & Absence & 99,130 & 76 & \multirow[t]{2}{*}{-} \\
\hline & $(3,456)$ & $(3.4)$ & Presence & 408 & 0 & \\
\hline \multirow[t]{2}{*}{ Stillbirth ( $\geq 12$ wk gestation) ${ }^{b}$} & 100,048 & 97.1 & Absence & 99,130 & 76 & \multirow[t]{2}{*}{0.478} \\
\hline & $(3,022)$ & $(2.9)$ & Presence & 841 & 1 & \\
\hline \multirow[t]{2}{*}{ Pre-term birth $<37$ wk gestation ${ }^{c}$} & 99,475 & 96.5 & Absence & 94,090 & 67 & \multirow[t]{2}{*}{0.020} \\
\hline & $(3,595)$ & $(3.5)$ & Presence & 5,309 & 9 & \\
\hline \multirow[t]{2}{*}{ Pre-term birth at $34-36$ wk gestation ${ }^{c}$} & 98,196 & 95.3 & Absence & 94,090 & 67 & \multirow[t]{2}{*}{0.539} \\
\hline & $(4,874)$ & $(4.7)$ & Presence & 4,035 & 4 & \\
\hline \multirow[t]{2}{*}{ Pre-term birth $<34$ wk gestation ${ }^{c}$} & 95,436 & 92.6 & Absence & 94,090 & 67 & \multirow[t]{2}{*}{0.003} \\
\hline & $(7,634)$ & $(7.4)$ & Presence & 1,274 & 5 & \\
\hline \multirow[t]{2}{*}{ Placenta praeviac } & 99,519 & 96.6 & Absence & 98,777 & 75 & \multirow[t]{2}{*}{0.400} \\
\hline & $(3,551)$ & $(3.4)$ & Presence & 666 & 1 & \\
\hline \multirow[t]{2}{*}{ Abruptio placentae $^{c}$} & 99,519 & 96.6 & Absence & 99,009 & 75 & \multirow[t]{2}{*}{0.283} \\
\hline & $(3,551)$ & $(3.4)$ & Presence & 434 & 1 & \\
\hline \multirow[t]{2}{*}{ Adherent placentac $^{c}$} & 99,519 & 96.6 & Absence & 99,209 & 76 & - \\
\hline & $(3,551)$ & $(3.4)$ & Presence & 234 & 0 & \\
\hline Premature rupture ${ }^{c}$ & 99,519 & 96.6 & Absence & 90,648 & 68 & 0.545 \\
\hline & $(3,551)$ & $(3.4)$ & Presence & 8,795 & 8 & \\
\hline Oligohydramnios $^{c}$ & 99,519 & 96.6 & Absence & 98,169 & 72 & 0.017 \\
\hline & $(3,551)$ & $(3.4)$ & Presence & 1,274 & 4 & \\
\hline Mild hypertensive disorders of pregnancy ${ }^{c}$ & 99,519 & 96.6 & Absence & 97,141 & 74 & 0.696 \\
\hline & $(3,551)$ & $(3.4)$ & Presence & 2,302 & 2 & \\
\hline Severe hypertensive disorders of pregnancy ${ }^{c}$ & 99,519 & 96.6 & Absence & 98,469 & 72 & 0.007 \\
\hline & $(3,551)$ & $(3.4)$ & Presence & 974 & 4 & \\
\hline Uterine infection & 100,818 & 97.8 & Absence & 100,008 & 75 & 0.109 \\
\hline & $(2,252)$ & $(2.2)$ & Presence & 733 & 2 & \\
\hline Caesarean section $^{c}$ & 99,165 & 96.2 & Vaginal & 79,785 & 49 & 0.001 \\
\hline & $(3,905)$ & $(3.8)$ & Caesarean & 19,304 & 27 & \\
\hline SFD $(<10 \%)^{c}$ & 99,360 & 96.4 & Absence & 89,385 & 69 & 1.000 \\
\hline & $(3,710)$ & $(3.6)$ & Presence & 9,899 & 7 & \\
\hline Multiple pregnancy & 100,733 & 97.7 & Single & 99,670 & 74 & 0.040 \\
\hline & $(2,337)$ & $(2.3)$ & Multiple & 986 & 3 & \\
\hline
\end{tabular}

Abbreviation: SFD, small for dates.

a'Stillbirth ( $\geq 12$ weeks' gestation) and artificial abortion were excluded from analyses.

'Early miscarriage ( $<12$ weeks' gestation) and artificial abortion were excluded from analyses.

${ }^{c}$ Miscarriage and artificial abortion were excluded from analyses.

${ }^{\mathrm{d}}$ The $p$-value was not calculated when expected frequency is less than 1 . 
New Risk Factors for VTE during Pregnancy and Post-Partum Sugiura-Ogasawara et al. 613

MTHFR and ANXA5 single-nucleotide polymorphisms is reported to be associated with RPL. ${ }^{20,21}$ Recent large cohort study proved that the rate of VTE within 42 days of an induced abortion was 3.0 per 10,000 women (hazard ratio,
$0.16,95 \% \mathrm{CI}, 0 \cdot 12-0 \cdot 22)$ when compared with women in the live birth cohort, whose VTE rate was 18.5 per 10,000 women. ${ }^{22}$ The risk might be due to pathologies common to RPL and VTE.

Table 3 An association of medical histories, gynaecological disease and lifestyle with venous thromboembolism

\begin{tabular}{|c|c|c|c|c|c|}
\hline & Factors & Crude ORs $(95 \% \mathrm{Cl})$ & $p$-Value & $\begin{array}{l}\text { Adjusted ORs } \\
(95 \% \mathrm{Cl})\end{array}$ & $p$-Value \\
\hline \multirow[t]{12}{*}{$\begin{array}{l}\text { Medical } \\
\text { histories }\end{array}$} & Atopic dermatitis & $\begin{array}{l}1.43 \\
(0.82-2.48)\end{array}$ & 0.204 & $\begin{array}{l}1.58 \\
(0.89-2.82)\end{array}$ & 0.119 \\
\hline & Asthma & $\begin{array}{l}1.53 \\
(0.83-2.83)\end{array}$ & 0.178 & $\begin{array}{l}1.55 \\
(0.81-2.96)\end{array}$ & 0.188 \\
\hline & Gestational diabetes & $\begin{array}{l}1.77 \\
(0.25-12.71)\end{array}$ & 0.573 & - & - \\
\hline & Hypothyroidism & $\begin{array}{l}1.34 \\
(0.19-9.61)\end{array}$ & 0.774 & - & - \\
\hline & Anaemia & $\begin{array}{l}1.18 \\
(0.68-2.04)\end{array}$ & 0.565 & $\begin{array}{l}1.22 \\
(0.68-2.21)\end{array}$ & 0.506 \\
\hline & Depression & $\begin{array}{l}1.78 \\
(0.65-4.87)\end{array}$ & 0.263 & - & - \\
\hline & Dysautonomia & $\begin{array}{l}1.45 \\
(0.53-3.97)\end{array}$ & 0.471 & - & - \\
\hline & Anxiety disorder & $\begin{array}{l}2.42 \\
(0.98-6.01)\end{array}$ & 0.056 & - & - \\
\hline & Gastritis & $\begin{array}{l}1.79 \\
(0.94-3.39)\end{array}$ & 0.074 & $\begin{array}{l}1.80 \\
(0.92-3.53)\end{array}$ & 0.089 \\
\hline & Gastric ulcer & $\begin{array}{l}2.36 \\
(0.74-7.48)\end{array}$ & 0.146 & - & - \\
\hline & Irritable colon & $\begin{array}{l}0.84 \\
(0.12-6.06)\end{array}$ & 0.865 & - & - \\
\hline & Cervical cancer & $\begin{array}{l}1.62 \\
(0.23-11.65)\end{array}$ & 0.633 & - & - \\
\hline \multirow[t]{7}{*}{$\begin{array}{l}\text { Pregnancy } \\
\text { histories }\end{array}$} & Pregnancy loss (total number) & $\begin{array}{l}1.83 \\
(1.14-2.92)\end{array}$ & 0.012 & $\begin{array}{l}1.42 \\
(0.84-2.39)\end{array}$ & 0.186 \\
\hline & Pregnancy loss (once) & $\begin{array}{l}1.30 \\
(0.73-2.32)\end{array}$ & 0.368 & $\begin{array}{l}1.11 \\
(0.59-2.06)\end{array}$ & 0.755 \\
\hline & Pregnancy loss (twice) & $\begin{array}{l}1.58 \\
(0.57-4.37)\end{array}$ & 0.382 & - & - \\
\hline & Pregnancy loss (three times or more) & $\begin{array}{l}10.92 \\
(5.16-23.08)\end{array}$ & $<0.001$ & $\begin{array}{l}6.13 \\
(2.48-15.16)\end{array}$ & $<0.001$ \\
\hline & Abruptio placentae & $\begin{array}{l}5.59 \\
(0.77-40.34)\end{array}$ & 0.088 & - & - \\
\hline & Ectopic pregnancy & $\begin{array}{l}1.38 \\
(0.19-9.90)\end{array}$ & 0.752 & - & - \\
\hline & Placenta praevia & $\begin{array}{l}5.60 \\
(1.37-22.88)\end{array}$ & 0.016 & - & - \\
\hline \multirow[t]{3}{*}{$\begin{array}{l}\text { Gynaecological } \\
\text { disease }\end{array}$} & Menstrual disorder & $\begin{array}{l}0.92 \\
(0.44-1.92)\end{array}$ & 0.829 & $\begin{array}{l}0.93 \\
(0.42-2.03)\end{array}$ & 0.851 \\
\hline & Endometriosis & $\begin{array}{l}2.68 \\
(1.23-5.83)\end{array}$ & 0.013 & $\begin{array}{l}2.70 \\
(1.21-6.00)\end{array}$ & 0.015 \\
\hline & Uterine fibroids & $\begin{array}{l}2.07 \\
(1.03-4.15)\end{array}$ & 0.041 & $\begin{array}{l}1.73 \\
(0.84-3.58)\end{array}$ & 0.139 \\
\hline
\end{tabular}

(Continued) 
Table 3 (Continued)

\begin{tabular}{|c|c|c|c|c|c|}
\hline & Factors & Crude ORs (95\% CI) & $p$-Value & $\begin{array}{l}\text { Adjusted } \mathrm{ORs}^{\mathrm{a}} \\
(95 \% \mathrm{Cl})\end{array}$ & $p$-Value \\
\hline & Adenomyosis & $\begin{array}{l}7.94 \\
(1.94-32.46)\end{array}$ & $<0.001$ & - & - \\
\hline & Ovarian tumour & $\begin{array}{l}0.75 \\
(0.19-3.07)\end{array}$ & 0.691 & - & - \\
\hline & Polycystic ovarian syndrome & $\begin{array}{l}3.07 \\
(1.24-7.61)\end{array}$ & 0.015 & - & - \\
\hline \multirow[t]{11}{*}{ Lifestyle } & Strong exercise during pregnancy & $\begin{array}{l}1.61 \\
(0.59-4.40)\end{array}$ & 0.356 & - & - \\
\hline & Moderate exercise during pregnancy & $\begin{array}{l}0.52 \\
(0.28-0.99)\end{array}$ & 0.046 & $\begin{array}{l}0.62 \\
(0.33-1.19)\end{array}$ & 0.154 \\
\hline & Walking during pregnancy & $\begin{array}{l}0.76 \\
(0.47-1.23)\end{array}$ & 0.264 & $\begin{array}{l}0.72 \\
(0.43-1.19)\end{array}$ & 0.199 \\
\hline & Night shift work during pregnancy & $\begin{array}{l}0.95 \\
(0.41-2.18)\end{array}$ & 0.898 & $\begin{array}{l}1.03 \\
(0.44-2.39)\end{array}$ & 0.954 \\
\hline & Breakfast during pregnancy & $\begin{array}{l}0.77 \\
(0.45-1.31)\end{array}$ & 0.330 & $\begin{array}{l}0.86 \\
(0.48-1.54)\end{array}$ & 0.607 \\
\hline & Working h/wk (MT1) (0 h vs. 1-35 h) & $\begin{array}{l}0.99 \\
(0.53-1.83)\end{array}$ & 0.968 & $\begin{array}{l}0.82 \\
(0.42-1.60)\end{array}$ & 0.562 \\
\hline & Working h/wk (MT1) (0 h vs. 36-45 h) & $\begin{array}{l}0.94 \\
(0.52-1.70)\end{array}$ & 0.831 & $\begin{array}{l}0.77 \\
(0.40-1.50)\end{array}$ & 0.443 \\
\hline & Working h/wk (MT1) (0 h vs. 46 h) & $\begin{array}{l}1.44 \\
(0.74-2.78)\end{array}$ & 0.280 & $\begin{array}{l}1.28 \\
(0.62-2.63)\end{array}$ & 0.500 \\
\hline & Working h/wk (MT2) (0 h vs. 1-35 h) & $\begin{array}{l}1.03 \\
(0.55-1.92)\end{array}$ & 0.937 & $\begin{array}{l}0.82 \\
(0.41-1.64)\end{array}$ & 0.575 \\
\hline & Working h/wk (MT2) (0 h vs. 36-45 h) & $\begin{array}{l}1.17 \\
(0.66-2.05)\end{array}$ & 0.591 & $\begin{array}{l}0.95 \\
(0.50-1.82)\end{array}$ & 0.884 \\
\hline & Working h/wk (MT2) (0 h vs. 46 h) & $\begin{array}{l}1.45 \\
(0.72-2.95)\end{array}$ & 0.301 & $\begin{array}{l}1.53 \\
(0.72-3.25)\end{array}$ & 0.274 \\
\hline
\end{tabular}

Abbreviations: BMI, body mass index; $\mathrm{Cl}$, confidence interval; IVF-ET, in vitro fertilization and embryo transfer; MT1, maternal questionnaires during the first trimester; MT2, maternal questionnaires during the second/third trimester; OR, odds ratio; VTE, venous thromboembolism.

${ }^{a}$ Adjusted for maternal age at registration, BMI, the presence/absence of IVF-ET, smoking and income. Only crude ORs were shown when the number of presence of both factor and VTE was less than 6 .

The frequency of VTE was found to be 7.5 per 10,000 pregnancies in the present Japanese population registered between 2011 and 2014. It was similar to that in Caucasian population, ${ }^{1}$ but VTE has been speculated to be less frequent in the Japanese population due to the fact that no FV Leiden and pro-thrombin mutations were found in the Japanese population. ${ }^{9}$ The frequency of PS deficiency was higher in the Japanese population because of a domestic mutation known as PS-Tokushima (K196E) which has a frequency of $1.8 \% .^{23}$

According to the old guidelines, thromboprophylaxis with the use of unfractionated heparin was speculated to be common for post-partum women with a previous episode of VTE or thrombophilia or for older obese women post-partum after a caesarean section that took place between 2011 and 2014. ${ }^{8}$ After April 2014, thromboprophylaxis during pregnancy was introduced into the JSOG guidelines. ${ }^{7}$ Thus, the effect of thromboprophylaxis during pregnancy might be small in this analysis, but we should consider that some portion of patients with an unexplained RPL may have received a combination of low dose aspirin and unfractionated heparin even though there was no recorded evidence. ${ }^{24}$ Actually, 267 and 1,609 women in the two groups above received heparin and low dose aspirin in the present cohort. However, any indication of treatment after VTE, prophylaxis for VTE or prevention of APS or gestational weeks at the start of heparin therapy was not available. This is one of the limitations of this study.

Associations with pre-term birth, HDP, uterine infection, multiple pregnancies and caesarean section with VTE were reported in the previous studies. ${ }^{2,3,5}$ A recent risk prediction model showed that emergency caesarean delivery, stillbirth, varicose veins, pre-eclampsia and post-partum infection were the strongest predictors of post-partum VTE. ${ }^{1}$ A pre-conceptional history of VTE was reportedly associated with an 
New Risk Factors for VTE during Pregnancy and Post-Partum Sugiura-Ogasawara et al. 615

Table 4 An association between pregnancy complication and venous thromboembolism

\begin{tabular}{|c|c|c|c|c|}
\hline Factors & $\begin{array}{l}\text { Crude ORs } \\
(95 \% \mathrm{Cl})\end{array}$ & p-Value & $\begin{array}{l}\text { Adjusted ORs }{ }^{\mathrm{a}} \\
(95 \% \mathrm{Cl})\end{array}$ & p-Value \\
\hline Threatened abortion & $\begin{array}{l}3.52 \\
(2.18-5.67)\end{array}$ & $<0.001$ & $\begin{array}{l}3.61 \\
(2.16-6.02)\end{array}$ & $<0.001$ \\
\hline Threatened pre-term labour & $\begin{array}{l}2.60 \\
(1.65-4.12)\end{array}$ & $<0.001$ & $\begin{array}{l}2.98 \\
(1.83-4.85)\end{array}$ & $<0.001$ \\
\hline Hyperemesis & $\begin{array}{l}1.23 \\
(0.65-2.34)\end{array}$ & 0.521 & $\begin{array}{l}1.21 \\
(0.62-2.38)\end{array}$ & 0.573 \\
\hline Stillbirth ( $\geq 12$ wk gestation) & $\begin{array}{l}1.55 \\
(0.22-11.17)\end{array}$ & 0.663 & - & - \\
\hline Pre-term birth $<37$ wk gestation ${ }^{b}$ & $\begin{array}{l}2.38 \\
(1.19-4.78)\end{array}$ & 0.015 & $\begin{array}{l}2.64 \\
(1.30-5.36)\end{array}$ & 0.007 \\
\hline Pre-term birth 34-36 wk gestation ${ }^{\mathrm{b}}$ & $\begin{array}{l}1.39 \\
(0.51-3.82)\end{array}$ & 0.521 & - & - \\
\hline Pre-term birth $<34$ wk gestation ${ }^{\mathrm{b}}$ & $\begin{array}{l}5.51 \\
(2.22-13.70)\end{array}$ & $<0.001$ & - & - \\
\hline Placenta praeviab & $\begin{array}{l}1.98 \\
(0.28-14.24)\end{array}$ & 0.499 & - & - \\
\hline Abruptio placentae ${ }^{b}$ & $\begin{array}{l}3.04 \\
(0.42-21.93)\end{array}$ & 0.270 & - & - \\
\hline Premature rupture ${ }^{b}$ & $\begin{array}{l}1.21 \\
(0.58-2.52)\end{array}$ & 0.606 & $\begin{array}{l}1.14 \\
(0.52-2.50)\end{array}$ & 0.744 \\
\hline Oligohydramnios $^{\mathrm{b}}$ & $\begin{array}{l}4.28 \\
(1.56-11.73)\end{array}$ & 0.005 & - & - \\
\hline Mild hypertensive disorders of pregnancy ${ }^{b}$ & $\begin{array}{l}1.14 \\
(0.28-4.65)\end{array}$ & 0.854 & - & - \\
\hline Severe hypertensive disorders of pregnancy ${ }^{b}$ & $\begin{array}{l}5.62 \\
(2.05-15.40)\end{array}$ & $<0.001$ & - & - \\
\hline Uterine infection & $\begin{array}{l}3.64 \\
(0.89-14.85)\end{array}$ & 0.072 & - & - \\
\hline Caesarean section $^{\mathrm{b}}$ & $\begin{array}{l}2.28 \\
(1.42-3.64)\end{array}$ & $<0.001$ & $\begin{array}{l}2.19 \\
(1.32-3.63)\end{array}$ & $<0.001$ \\
\hline SFD $(<10 \%)^{b}$ & $\begin{array}{l}0.92 \\
(0.42-1.99)\end{array}$ & 0.825 & $\begin{array}{l}0.95 \\
(0.41-2.20)\end{array}$ & 0.899 \\
\hline Multiple pregnancy & $\begin{array}{l}4.10 \\
(1.29-13.02)\end{array}$ & 0.017 & - & - \\
\hline
\end{tabular}

Abbreviations: BMI, body mass index; Cl, confidence interval; IVF-ET, in vitro fertilization and embryo transfer; OR, odds ratio; SFD, small for dates; VTE, venous thromboembolism.

${ }^{a}$ Adjusted for maternal age at registration, BMI, the presence/absence of IVF-ET, smoking and income. Only crude ORs were shown when the number of presence of both factor and VTE was less than 6.

${ }^{\mathrm{b}}$ Miscarriage and artificial abortion were excluded from analyses.

increased risk of pre-eclampsia, stillbirth and placental abruption. ${ }^{25}$ These might also be induced by common genetic factors.

An association with oligohydramnios was not confirmed by a logistic model because of the small sample size. The major limitation was that there was no distinction made between VTE during pregnancy and that occurring post-partum. Data on gestational weeks at the VTE occurrence were also not available. This might be a reason why hyperemesis were not associated with VTE in this study. Thus, we could not dismiss these associations. The majority of women were recruited at 14 weeks' gestation, therefore, we should keep in mind that we did not cover all early miscarriages or hyperemesis.

Endometriosis and a history of RPL were found to be novel risk factors for VTE. Endometriosis and RPL affect 6 to $10 \%$ and $4.2 \%$ of women of reproductive age, respectively. ${ }^{16,26}$ Consequently, we should pay careful attention to novel predictors, such as endometriosis and RPL, to prevent VTE in pregnant women. 


\section{What is known about this topic?}

- Venous thromboembolism (VTE) prophylaxis during pregnancy and post-partum is recommended for women in high-risk groups.

- The strongest risk factor is a previous episode. Thrombophilia, an age $\geqq 35$ years, obesity, a smoker, parity $\geqq 3$, a systemic infection, gross varicose veins, paraplegia, pre-eclampsia, hyperemesis, multiple pregnancies, caesarean section, stillbirth and post-partum haemorrhage were considered to be risk factors.

- The number of large population-based studies examining predictors for VTE has been limited, and there has been no study based on a Japanese population.

\section{What does this paper add?}

- Endometriosis and recurrent pregnancy loss (RPL) were identified as novel independent risk factors for VTE.

- Threatened abortion, threatened pre-term birth, preterm birth and caesarean section were ascertained to be risk factors for VTE.

\section{Authors' Contributions}

The JECS group conducted the nationwide study project. M.S.O. designed the present study, analysed the data and wrote the first draft of the manuscript. T.E. organized the study team and was responsible for obtaining and analysing the data. M.K., a member of the JECS Steering Committee, was responsible for data acquisition and supervision of the study. M.K. and T.O. took the initiative in the launch of the Aichi regional sub-cohort of JECS. T.M. analysed the data. Y.Y., T.O., Y.M., S.K. and S.S. were responsible for data acquisition. All authors interpreted the data, contributed to the writing of the manuscript and revised it critically for important intellectual content.

\section{Note}

Data sharing is not permitted by the JECS due to a government policy restricting the deposition of data containing personal information. See the reference for more details. ${ }^{12}$

\section{Funding}

This study was funded by the Ministry of the Environment, Government of Japan.

\section{Conflict of Interest}

M.S.-O. received grants from the Japanese Ministry of Education, Science, and Technology for conducting studies on the topic of RPL in the different patient population, and payment for lectures from Kaken Pharmaceutical Co. Ltd., Kissei Pharmaceutical Co., Aska Pharmaceutical Co. Ltd., Sekisui Medical Co. Ltd. and Siemens Japan. The remaining authors report no conflict of interest.

\section{Acknowledgements}

We would like to express our gratitude to all of the JECS participants and all obstetricians in the 15 areas.

The findings and conclusions of this article are solely the responsibility of the authors and do not represent the official views of the Ministry of the Environment.

The chief members of the Japan Environment and Children's Study (JECS) as of 2017 are the National Institute for Environmental Studies, Tsukuba (principal investigator, Toshihiro Kawamoto); the National Center for Child Health and Development, Tokyo (Hirohisa Saito); Hokkaido University, Sapporo (Reiko Kishi); Tohoku University, Sendai (Nobuo Yaegashi); Fukushima Medical University, Fukushima (Koichi Hashimoto); Chiba University, Chiba (Chisato Mori); Yokohama City University, Yokohama (Shuichi Ito); University of Yamanashi, Chuo (Zentaro Yamagata); University of Toyama, Toyama (Hidekuni Inadera); Nagoya City University, Nagoya (Michihiro Kamijima); Kyoto University, Kyoto (Takeo Nakayama); Osaka University, Suita (Hiroyasu Iso); Hyogo College of Medicine, Nishinomiya (Masayuki Shima); Tottori University, Yonago (Yasuaki Hirooka); Kochi University, Nankoku (Narufumi Suganuma), University of Occupational and Environmental Health, Kitakyushu (Koichi Kusuhara); and Kumamoto University, Kumamoto (Takahiko Katoh).

\section{References}

1 Sultan AA, West J, Grainge MJ, et al. Development and validation of risk prediction model for venous thromboembolism in postpartum women: multinational cohort study. BMJ 2016;355:i6253

2 Bates SM, Greer IA, Middeldorp S, Veenstra DL, Prabulos AM, Vandvik PO. Venous thromboembolism, thrombophilia, antithrombotic therapy, and pregnancy. American College of Chest Physicians Evidence-Based Clinical Practice Guidelines (9th Edition). Chest 2012;141:691-736

3 Royal College of Obstet Gynecol. Reducing the risk of thrombosis and embolism during pregnancy and the puerperium. RCOG Green-top Guideline No. 37a;2015

4 Brill-Edwards P, Ginsberg JS, Gent M, et al. Recurrence of Clot in This Pregnancy Study Group. Safety of withholding antepartum heparin in women with a previous episode of venous thromboembolism. N Engl J Med 2000;343:1439-1444

5 Sultan AA, Tata LJ, West J, et al. Risk factors for first venous thromboembolism around pregnancy: a population-based cohort study from the United Kingdom. Blood 2013;121(19):3953-3961

6 Bain E, Wilson A, Tooher R, Gates S, Davis LJ, Middleton P. Prophylaxis for venous thromboembolic disease in pregnancy and the early postnatal period. Cochrane Database Syst Rev 2014; 2(02):CD001689

7 Minakami H, Maeda T, Fujii T, et al. Guidelines for obstetrical practice in Japan: Japan Society of Obstetrics and Gynecology (JSOG) and Japan Association of Obstetricians and Gynecologists (JAOG) 2014 edition.J Obstet Gynaecol Res 2014;40(06):1469-1499 8 Editorial Committee on Japanese Guidelines for Prevention of Venous Thromboembolism. Japanese guidelines for prevention of venous thromboembolism. Tokyo, Japan: Medical Front International Limited; 2004:47-54

9 James AH, Jamison MG, Brancazio LR, Myers ER. Venous thromboembolism during pregnancy and the postpartum period: incidence, risk factors, and mortality. Am J Obstet Gynecol 2006;194 (05):1311-1315

10 Kawamoto T, Nitta H, Murata K, et al; Working Group of the Epidemiological Research for Children's Environmental Health. 
Rationale and study design of the Japan Environment and Children's Study (JECS). BMC Public Health 2014;14:25

11 Michikawa T, Nitta H, Nakayama SF, et al; Japan Environment and Children's Study Group. Baseline profile of participants in the Japan Environment and Children's Study (JECS). J Epidemiol 2018; 28(02):99-104

12 Suzuki K, Shinohara R, Sato M, Otawa S, Yamagata Z. Association between maternal smoking during pregnancy and birth weight: an appropriately adjusted model from the Japan Environment and Children's Study. J Epidemiol 2016;26(07):371-377

13 Japan Environment and Children's Study. Tokyo: Ministry of the Environment. Available at: http://www.env.go.jp/en/chemi/hs/ jecs/ [updated August 12, 2016]. Accessed August 16, 2016

14 Ishitsuka K, Nakayama SF, Kishi R, et al. Japan Environment and Children's Study: backgrounds, activities, and future directions in global perspectives. Environ Health Prev Med 2017;22(01):61

15 National Institute for Environmental Studies/National Centre for Japan Environment and Children's Study, Japan Environment and Children's Study (JECS) Study Protocol (ver. 1.4). Available at: https://www.env.go.jp/chemi/ceh/outline/data/jecs-study_proto col_14_en.pdf. Accessed January 18, 2017

16 Giudice LC. Clinical practice. Endometriosis. N Engl J Med 2010; 362(25):2389-2398

17 Ageno W, Manfredi E, Dentali F, et al. The incidence of venous thromboembolism following gynecologic laparoscopy: a multicenter, prospective cohort study. J Thromb Haemost 2007;5(03): 503-506

18 Croles FN, Nasserinejad K, Duvekot JJ, Kruip MJ, Meijer K, Leebeek FW. Pregnancy, thrombophilia, and the risk of a first venous thrombosis: systematic review and Bayesian meta-analysis. BMJ 2017;359:j4452

19 Miyakis S, Lockshin MD, Atsumi T, et al. International consensus statement on an update of the classification criteria for definite antiphospholipid syndrome (APS). J Thromb Haemost 2006;4 (02):295-306

20 Pereza N, Ostojić S, Kapović M, Peterlin B. Systematic review and meta-analysis of genetic association studies in idiopathic recurrent spontaneous abortion. Fertil Steril 2017;107(01):150-159

21 Hayashi Y, Sasaki H, Suzuki S, et al. Genotyping analyses for polymorphisms of ANXA5 gene in patients with recurrent pregnancy loss. Fertil Steril 2013;100(04):1018-1024

22 Liu N, Vigod SN, Farrugia MM, Urquia ML, Ray JG. Venous thromboembolism after induced abortion: a population-based, propensity-score-matched cohort study in Canada. Lancet Haematol 2018;5(07):e279-e288

23 Kimura R, Honda S, Kawasaki T, et al. Protein S-K196E mutation as a genetic risk factor for deep vein thrombosis in Japanese patients. Blood 2006;107(04):1737-1738

24 Kaandorp SP, Goddijn M, van der Post JAM, et al. Aspirin plus heparin or aspirin alone in women with recurrent miscarriage. N Engl J Med 2010;362(17):1586-1596

25 Hansen AT, Schmidt M, Horváth-Puhó E, et al. Preconception venous thromboembolism and placenta-mediated pregnancy complications. J Thromb Haemost 2015;13(09):1635-1641

26 Sugiura-Ogasawara M, Suzuki S, Ozaki Y, Katano K, Suzumori N, Kitaori T. Frequency of recurrent spontaneous abortion and its influence on further marital relationship and illness: the Okazaki Cohort Study in Japan. J Obstet Gynaecol Res 2013;39(01):126-131 